Published in final edited form as:

Org Lett. 2021 February 05; 23(3): 777-780. doi:10.1021/acs.orglett.0c04001.

\title{
Amphiphilic Biaryl Monophosphine Ligands by Regioselective Sulfonation
}

\author{
Jacob Rodriguez ${ }^{\ddagger}$, Heemal H. Dhanjee ${ }^{\ddagger}$, Stephen L. Buchwald ${ }^{*}$ \\ Department of Chemistry, Massachusetts Institute of Technology, Cambridge, Massachusetts, \\ 02139, United States
}

\begin{abstract}
Amphiphilic ligands are valued for their ability to facilitate organometallic reactions in the presence of water. The regioselective sulfonation of a series of commercially available biaryl monophosphines to generate amphiphilic ligands is presented. In this one-step protocol, the temperature and addition of fuming sulfuric acid were carefully controlled to arrive at sulfonated biaryl monophosphine ligands in high yields with $>95 \%$ regioselectivity without the need for chromatographic purification.
\end{abstract}

\section{Graphical Abstract}

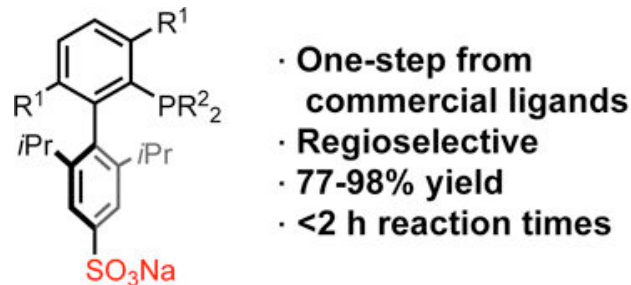

Sulfonated $t$-BuXPhos, BrettPhos, $t$-BuBrettPhos New protocols to prepare sulfonated SPhos, XPhos<smiles>COc1c(S(=O)(=O)O)cc(S(=O)(=O)O)c(OC)c1-c1ccccc1[18O]</smiles>

bis-sulfonated SPhos (bsSPhos)

high water-solubility

The use of water-soluble organometallic complexes often capitalizes on reaction rate acceleration due to hydrophobic clustering of substrates and lowering of transition state barriers through hydrogen bonding. ${ }^{1,2}$ Conducting reactions in aqueous conditions can also simplify the purification of products and ease recycling of catalytic complexes in biphasic solvent mixtures. However, many organometallic transformations are developed in organic

\footnotetext{
*Corresponding Author: Stephen L. Buchwald - Department of Chemistry, Massachusetts Institute of Technology, Cambridge, Massachusetts 02139, United States, sbuchwal@ mit.edu.

¥These authors contributed equally.

Jacob Rodriguez - Department of Chemistry, Massachusetts Institute of Technology, Cambridge, Massachusetts 02139, United States Heemal H. Dhanjee - Department of Chemistry, Massachusetts Institute of Technology, Cambridge, Massachusetts 02139, United States

The manuscript was written through contributions of all authors. All authors have given approval to the final version of the manuscript. Supporting Information

The Supporting Information is available free of charge on the ACS Publications website.

Experimental procedures and characterization data for all compounds (PDF)

The authors declare the following competing financial interest(s): MIT has or has filed patents on the ligands that are described in the paper from which S.L.B. and former coworkers receive royalty payments.
} 
solvents, and the metal-complexes employed are either minimally or completely insoluble in water. To adapt these transformations for use in an aqueous solvent without the need to employ surfactants or micelles, modification of the ancillary ligand is necessary. These changes to the ligand are designed to impart increased hydrophilicity of the resulting organometallic complex allowing for the corresponding organic transformations to be carried out in aqueous solvent. ${ }^{3,4}$

With regard to palladium-mediated transformations, biaryl monophosphines are a class of ligands that excel in promoting various $\mathrm{C}-\mathrm{C}$ and $\mathrm{C}$-heteroatom bond forming reactions. ${ }^{5}$ The substituents of each of these ligands has been designed to facilitate key elements of the Pd-mediated cross-coupling catalytic cycle, including oxidative addition and reductive elimination. ${ }^{6}$ However, hydrophilic analogues of this particular class of ligands are largely absent from the literature. Sulfonation of the ancillary ligand imparts increased water solubility to the resulting organopalladium complex, expanding its effective reactivity to hydrophilic substrates. In addition to aqueous solubility considerations, amphiphilic biaryl monophosphine ligands have found utility in electrostatically guided Pd-catalysis. ${ }^{7,8}$ Our interest in these ligands stemmed from our ongoing efforts in the use of organometallic palladium reagents for bioconjugation where biopolymer substrates such as proteins often require the use of water as a solvent to prevent denaturation. ${ }^{9}$

Our group has previously described mild heteroatom-arylation reactions for the conjugation of lysine and cysteine residues utilizing stoichiometric palladium oxidative addition complexes (OACs) of the type [ $\mathrm{LPd}(\mathrm{Ar}) \mathrm{X}]\left(\mathrm{L}=\right.$ biaryl monophosphine, $\mathrm{X}=\mathrm{Cl}, \mathrm{Br}$, OTf) ${ }^{10}$ For these reactions, ligand choice was a key parameter that influenced chemoselectivity ${ }^{11}$ and overall yield. ${ }^{12}$ Sulfonated SPhos (sSPhos, 2), previously prepared via sulfonation of SPhos $(\mathbf{1})^{13}$ (Figure 1A), allowed us to carry out $S$-arylation with enhanced efficiency in aqueous solutions presumably due to the increased water solubility imparted onto the OAC reagent. ${ }^{14}$ While 2 confers greater water solubility on what would otherwise be a hydrophobic complex, most OACs we prepared were only soluble at micromolar concentrations and, in some cases, still required the use of an organic co-solvent. ${ }^{15}$

To improve the water solubility of Pd-OACs derived from biaryl monophosphine ligands known to accommodate $N-, O$ - and $S$-nucleophiles, we set out to prepare sulfonated variants of commercially available biaryl monophosphine ligands. Here we disclose new protocols for the preparation of $\mathbf{2}$ and sXPhos $(\mathbf{5})^{13}$ (Figure 1A, B) that is complete within hours or, in most cases, minutes. These improved protocols are further applied for the sulfonation of four commercially available biaryl monophosphine ligands to give the corresponding sulfonated ligands in a single synthetic step, each prepared on a one-gram scale (Figure 1C).

During the preparation of $\mathbf{2}$ using our literature protocol (Fig. 1A), ${ }^{13}$ monitoring of the reaction by tandem liquid-chromatography mass spectrometry (LC-MS) showed that the conversion of $\mathbf{1}$ to its sulfonated form $\mathbf{2}$ was complete at room temperature in less than two hours, not requiring the elevated temperature $\left(40^{\circ} \mathrm{C}\right)$ or $24 \mathrm{~h}$ reaction time reported. Isolation and analysis of the product after a one-hour reaction time confirmed its identity as 2 with ${ }^{1} \mathrm{H},{ }^{13} \mathrm{C}$ and ${ }^{31} \mathrm{P}$ NMR spectra comparable to that reported in the literature. 
Given the rapid sulfonation we observed for ligand 1, we investigated whether other commercially available ligands would maintain a similarly high reactivity. The original protocol reported by our lab $^{13}$ and repeated by others ${ }^{8}$ for the sulfonation of $\mathbf{1}$ also detailed a sulfonation strategy for the selective monosulfonation of XPhos (3) via a Friedel-Crafts/ retro-Friedel-Crafts reaction (Figure 1B). We therefore examined this approach toward the sulfonation of BrettPhos (10, Scheme 1) which has not previously been reported to undergo sulfonation. Unlike $\mathbf{1}$ or $\mathbf{3}, \mathbf{1 0}$ contains an electron-rich top ring bearing two electrondonating methoxy groups. It was unclear if or where sulfonation would occur, but we hypothesized that protonation of the phosphine under the reaction conditions would deactivate the top ring toward electrophilic aromatic substitution. Thus, we employed conditions analogous to those used in the preparation of $\mathbf{5}$. First, $\mathbf{1 0}$ was exposed to a mixture of $\mathrm{CH}_{2} \mathrm{Cl}_{2}$ and $\mathrm{H}_{2} \mathrm{SO}_{4}$ to protonate the phosphine followed by the addition of fuming sulfuric acid at $0{ }^{\circ} \mathrm{C}$. This gave a monosulfonated form of BrettPhos (sBrettPhos, 7) in $84 \%$ yield and proceeded to completion within minutes, validating the high reactivity of these biaryl systems toward sulfonation.

Next, we attempted an analogous protocol using $t$-BuBrettPhos (11, Table 1) as the substrate. Unfortunately, multiple products were observed when monitoring the reaction by LC-MS: two peaks with different retention times and the same $\mathrm{m} / \mathrm{z}$ corresponding to monosulfonated products (523 Da, entry 1). This observation was confirmed by ${ }^{1} \mathrm{H}$ and ${ }^{31} \mathrm{P}$ NMR analysis of the crude reaction mixture and indicated a mixture of isomers $\mathbf{8}$ and $\mathbf{1 2}$ in a ratio of 13:1, respectively. ${ }^{16}$ Although separation of the isomers was possible, we sought to optimize the regioselectivity of the sulfonation reaction to avoid any additional purification steps. We note that our initial attempts to prepare $\mathbf{5}$ and st-BuXPhos (6) also gave mixtures of unassigned products under analogous reaction conditions. We chose to continue optimizing the reaction using $\mathbf{1 1}$ as the substrate for our further studies.

From the outset of our optimization, it was unclear which parameters would most influence product distribution. Hypothesizing that the concentration of SO3 played an important role in the reaction, we reversed the order of addition of the reagents, adding a solution of $\mathbf{1 1}$ dropwise into fuming sulfuric acid. From this adjustment, a 48:1 ratio of 8:12 (entry 2) was obtained as determined by analysis of the crude reaction mixture by ${ }^{31} \mathrm{P}$ NMR. When this protocol was carried out at a measured bath temperature of $-10{ }^{\circ} \mathrm{C}$, the ratio of $8: 12$ formed was improved to $82: 1$ (entry 3 ).

With an optimized procedure for the selective monosulfonation of $\mathbf{1 1}$ on a 50 milligram scale, we extended this protocol to other biarylphosphine ligands and conducted it on a 1.0 gram scale (Scheme 2, $\mathbf{1 3}$ to $\mathbf{1 4}$ ). In each case, the sulfonated products 5, 6, and $\mathbf{8}$ were isolated with $>96: 4$ product distribution as assessed by ${ }^{31} \mathrm{P}$ NMR and in good yield (77\% $98 \%) \cdot{ }^{17}$

The high degree of reactivity of these ligands toward sulfonation caused us to then consider the use of fuming sulfuric acid to obtain a bis-sulfonated derivative of the more electron-rich 1 (bsSPhos, 9, Scheme 3). Thus, sequential treatment of SPhos with $\mathrm{H}_{2} \mathrm{SO}_{4}$ followed by the addition of fuming sulfuric acid (21-30\% $\mathrm{SO}_{3}$ basis) led to full conversion of $\mathbf{1}$ to $\mathbf{9}$, as determined by LC-MS. 
The increased water solubility of the deprotonated form of bsSPhos made extraction with organic solvents difficult. All previous efforts in our group for the preparation of sulfonated ligands showed that the products could be isolated by neutralization of the reaction mixture with aqueous $\mathrm{NaOH}$ followed by extraction with dichloromethane. As an alternative, we employed a method reported for the preparation of a bis-sulfonated version of XantPhos. ${ }^{18}$ Isolation of 9 was achieved by precipitating it through the addition of a controlled amount of water prior to neutralization, which provided the product in $96 \%$ yield as a single regioisomer. The addition of stoichiometric quantities of $\mathrm{NaOH}$ to generate the sodium salt of the product followed by lyophilization produced an unstable form of the ligand which decomposed over the course of several weeks when left on the bench, open to air. As a means to circumvent this issue, we have found that we can store the compound in its zwitterionic form. In this form, $\mathbf{9}$ was stable for six months under ambient conditions as indicated by ${ }^{1} \mathrm{H}$ NMR and ${ }^{31} \mathrm{P}$ NMR.

In summary, we have developed a modified sulfonation protocol for the controlled, regioselective sulfonation of the commercially available ligands SPhos, XPhos, $t$-BuXPhos, BrettPhos, and $t$-BuBrettPhos. ${ }^{19}$ Additionally, we have devised a protocol to prepare the bissulfonated version of SPhos, bsSPhos. In the case of bsSPhos, we anticipate the ligand to confer increased water solubility to what would otherwise be a hydrophobic complex, thus avoiding the need to modify the hydrophobic aryl halide electrophile for aqueous conjugation, with the ligand operating as a traceless solubility modifier. We expect these ligands will find use in bioconjugation, catalyst separation, and cation-pair directed Pd catalysis. $^{20}$

\section{Supplementary Material}

Refer to Web version on PubMed Central for supplementary material.

\section{ACKNOWLEDGMENT}

This work was supported by the NIH (Grant No. R35GM122483). We thank MilliporeSigma for the generous donation of all ligands used in this study. We also thank Alexander W. Schuppe (MIT) and Christine Nguyen (MIT) for advice and assistance in the preparation of this manuscript. This work was in part supported by an NIH postdoctoral fellowship under Grant No. 1F32GM131592-01A1 (H.H.D.). J. R. gratefully acknowledges support from the National Science Foundation Graduate Research Fellowship under Grant No. 174530 and the MIT Dean of Science Fellowship.

\section{REFERENCES}

(1). Shaughnessy KH Beyond TPPTS: New Approaches to the Development of Efficient PalladiumCatalyzed Aqueous-Phase Cross-Coupling Reactions. Eur. J. Org. Chem 2006, 8, 1827-1835.

(2). Butler RN; Coyne AG Water: Nature's Reaction Enforcer-Comparative Effects for Organic Synthesis "In-Water" and “On-Water." Chem. Rev 2010, 110, 6302-6337. [PubMed: 20815348]

(3). Cho JH; Prickett CD; Shaughnessy KH Efficient Sonogashira Coupling of Unprotected Halonucleosides in Aqueous Solvents Using Water-Soluble Palladium Catalysts. Eur. J. Org. Chem 2010, 19, 3678-3683.

(4). Messina MS; Maynard HD Modification of Proteins Using Olefin Metathesis. Mater. Chem. Front 2020, 4, 1040-1051.

(5). Stradiotto M, Lundgren RJ, Eds. Ligand Design in Metal Chemistry: Reactivity and Catalysis, 1st ed.; John Wiley \& Sons, Ltd: Chichester, UK, 2016. 
(6). Ingoglia BT; Wagen CC; Buchwald SL Biaryl Monophosphine Ligands in Palladium-Catalyzed C-N Coupling: An Updated User's Guide. Tetrahedron 2019, 75, 4199-4211. [PubMed: 31896889]

(7). (a)Golding WA; Phipps RJ Electrostatically-Directed Pd-Catalysis in Combination with C-H Activation: Site-Selective Coupling of Remote Chlorides with Fluoroarenes and Fluoroheteroarenes. Chem. Sci 2020, 11, 3022-3027.(b)Golding WA; Pearce-Higgins R; Phipps RJ Site-Selective Cross-Coupling of Remote Chlorides Enabled by Electrostatically Directed Palladium Catalysis. J. Am. Chem. Soc 2018, 140, 13570-13574. [PubMed: 30295472] (c)Golding WA; Schmitt HL; Phipps RJ Systematic Variation of Ligand and Cation Parameters Enables Site-Selective C-C and C-N Cross-Coupling of Multiply Chlorinated Arenes through Substrate-Ligand Electrostatic Interactions. J. Am. Chem. Soc 2020, 142, 21891-21898. [PubMed: 33332114]

(8). Thomas GT; Janusson E; Zijlstra HS; McIndoe JS Step-by-Step Real Time Monitoring of a Catalytic Amination Reaction. Chem. Commun 2019, 55, 11727-11730.

(9). Mattos C; Ringe D Proteins in Organic Solvents. Curr. Opin. Struct. Biol 2001, 11, 761-764. [PubMed: 11751059]

(10). Vinogradova EV; Zhang C; Spokoyny AM; Pentelute BL; Buchwald SL Organometallic Palladium Reagents for Cysteine Bioconjugation. Nature 2015, 526, 687-691. [PubMed: 26511579]

(11). Lee HG; Lautrette G; Pentelute BL; Buchwald SL Palladium-Mediated Arylation of Lysine in Unprotected Peptides. Angew. Chem. Int. Ed 2017, 56, 3177-3181.

(12). Zhao W; Lee HG; Buchwald SL; Hooker JM Direct ${ }^{11} \mathrm{CN}$-Labeling of Unprotected Peptides via Palladium-Mediated Sequential Cross-Coupling Reactions. J. Am. Chem. Soc 2017, 139, 71527155. [PubMed: 28502164]

(13). Anderson KW; Buchwald SL General Catalysts for the Suzuki-Miyaura and Sonogashira Coupling Reactions of Aryl Chlorides and for the Coupling of Challenging Substrate Combinations in Water. Angew. Chem. Int. Ed 2005, 44, 6173-6177.

(14). Rojas AJ; Pentelute BL; Buchwald SL Water-Soluble Palladium Reagents for Cysteine $S$ Arylation under Ambient Aqueous Conditions. Org. Lett 2017, 19, 4263-4266. [PubMed: 28777001]

(15). Dhanjee HH; Saebi A; Buslov I; Loftis AR; Buchwald SL; Pentelute BL Protein-Protein CrossCoupling via Palladium-Protein Oxidative Addition Complexes from Cysteine Residues. J. Am. Chem. Soc 2020, 142, 9124-9129. [PubMed: 32364380]

(16). For structural assignment of the major component of the mixture of isomers 12 by 1-D and 2-D NMR, see the Supporting Information (compound SI1)12SI1.

(17). To minimize the formation of phosphine oxide it was necessary to ensure that substrates were both: (1) fully dissolved in $\mathrm{CH}_{2} \mathrm{Cl}_{2}$ when cooled to $0{ }^{\circ} \mathrm{C}$ and (2) treated with sulfuric acid to protonate the phosphine and thereby protect it from oxidation prior to exposure to $\mathrm{SO}_{3}$. The lone exception to this was $\mathbf{1 5}$ where sulfuric acid was avoided; in short, we used a modified protocol to prevent significant (we observed 2-7\%) phosphine oxide formation. For more details, see the Supporting Information 22315

(18). Mul WP; Ramkisoensing K; Kamer PCJ; Reek JNH; van der Linden AJ; Marson A; van Leeuwen PWNM New, Highly Efficient Work-Up Protocol for Sulfonated Diphosphines. Adv. Synth. Catal 2002, 344, 293-298.

(19). We observed a higher degree of solubility for each of the sulfonated ligands in polar organic solvents. For further details see the Supporting Information.

(20). For a preprint of this paper, see: Rodriguez Jacob; Dhanjee Heemal; Buchwald Stephen L. Amphiphilic Biaryl Monophosphine Ligands by Regioselective Sulfonation. ChemRxiv 2020, DOI: 10.26434/chemrxiv.13337471.v1. 
A New, rapid protocol for the synthesis of a water-soluble ligand sSPhos:<smiles>COc1cccc(OC)c1-c1ccccc1P(=O)(O)O</smiles>

SPhos (1)

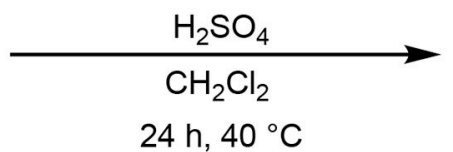

(99\% yield, ref. 11

this work: $1 \mathrm{~h}$, room temp., $85 \%$ yield)

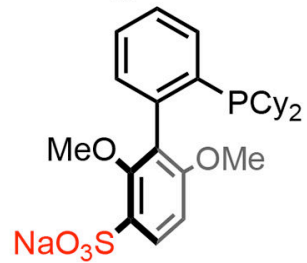

Sulfonated SPhos (sSPhos, 2)

B Sulfonation of XPhos via Friedel-Crafts/Retro-Friedel-Crafts:<smiles>CC(C)c1cc(C(C)C)c(-c2ccccc2P(C)(F)(F)F)c(C(C)C)c1</smiles>

XPhos (3)

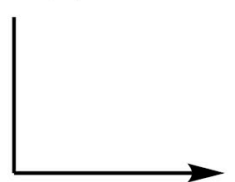

$\mathrm{H}_{2} \mathrm{SO}_{4}\left(30 \% \mathrm{SO}_{3}\right.$ basis $)$

$\mathrm{CH}_{2} \mathrm{Cl}_{2}, 24 \mathrm{~h}$

then aq. $\mathrm{NaOH}$

53-94\% yield (ref. 8,11) (this work: $77 \%$ yield, $5 \mathrm{~min}$ )

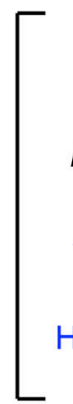

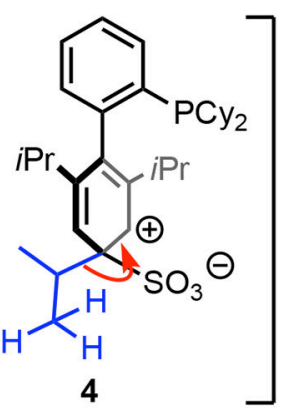

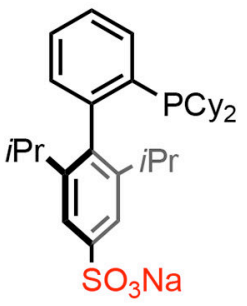
sXPhos (5)

C This work:

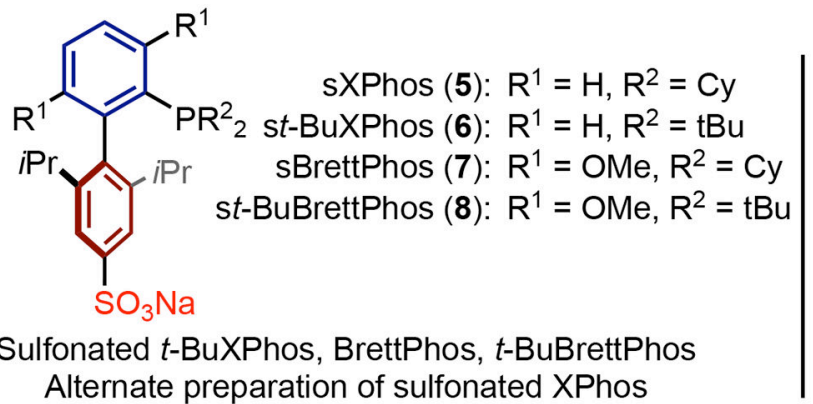<smiles>COc1cc([N+](=O)[O-])cc([18OH])c1-c1ccccc1[18O]</smiles>

bis-sulfonated SPhos (bsSPhos, 9)

Figure 1.

Preparation of sulfonated biaryl monophosphine ligands via sulfonation. (A) New protocols for the preparation of sulfonated SPhos (sSPhos, 2) and (B) sulfonated XPhos (sXPhos, 5) with reduced reaction times. (C) Synthesis of sulfonated $t$-BuXPhos, BrettPhos, and $t$ BuBrettPhos (st-BuXPhos (6), sBrettPhos (7), and st-BuBrettPhos (8) respectively). 


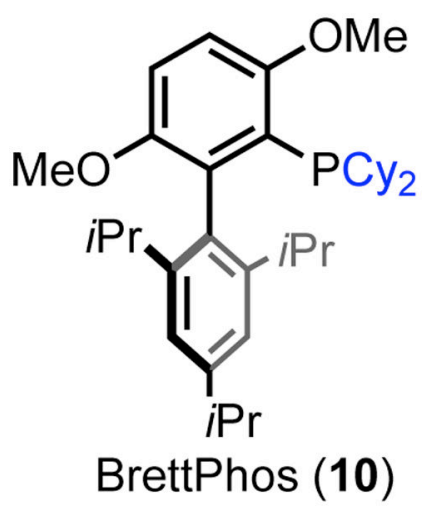

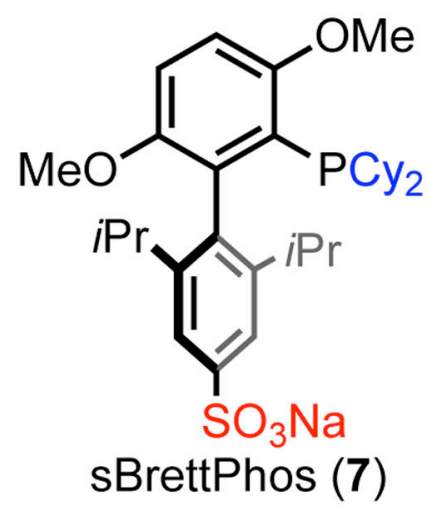

Scheme 1.

Monosulfonation of BrettPhos to provide sBrettPhos.

$\mathrm{CH}_{2} \mathrm{Cl}_{2}, \mathrm{H}_{2} \mathrm{SO}_{4}$ then $\mathrm{H}_{2} \mathrm{SO}_{4}\left(20-30 \% \mathrm{SO}_{3}\right)$

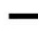

then aq. $\mathrm{NaOH}$

$84 \%$ yield 


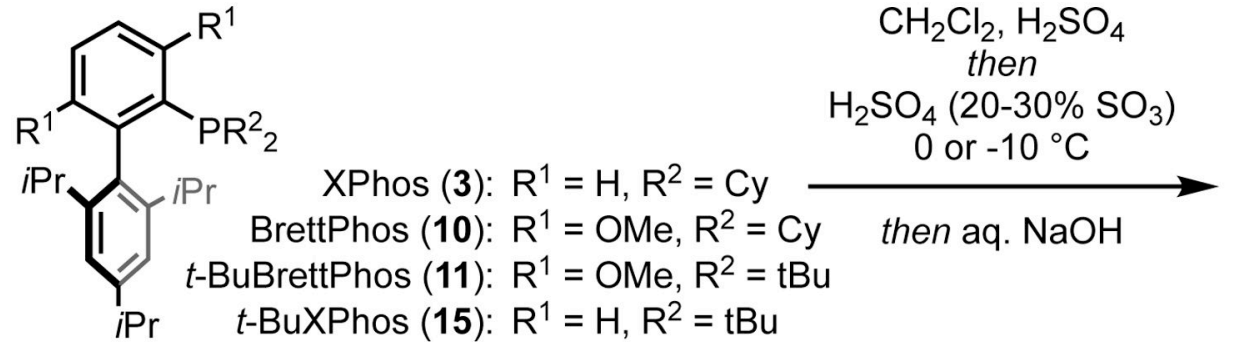

$13(1.0 \mathrm{~g})$<smiles>CCCc1cc(S(=O)(=O)O)cc(I)c1-c1ccccc1P(C)(=O)[O-]</smiles>

sXPhos (5)

$77 \%$ yield
XPhos (3): $R^{1}=H, R^{2}=C y$

(10): $R^{1}=O M e, R^{2}=C y$

(15): $R^{1}=H, R^{2}=t B u$

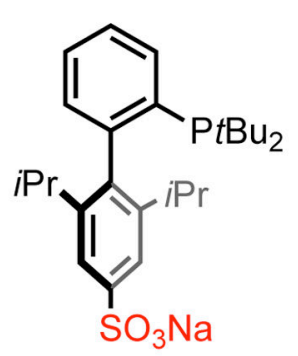<smiles>COc1ccc(OC)c(C(C)C)c1-c1c(F)cc([S+]([O])([O-])O)cc1I</smiles>

sBrettPhos (7) $84 \%$ yield<smiles>[R]c1ccc([R])c(-c2c([IH+])cc(S(=O)(=O)[O-])cc2[IH+])c1[R2]</smiles>

14<smiles>CCCCCCCC(C)C</smiles>

st-BuBrettPhos (8)

$98 \%$ yield

Scheme 2.

Gram-scale synthesis of sulfonated XPhos, $t$-BuXPhos, BrettPhos, and $t$-BuBrettPhos 


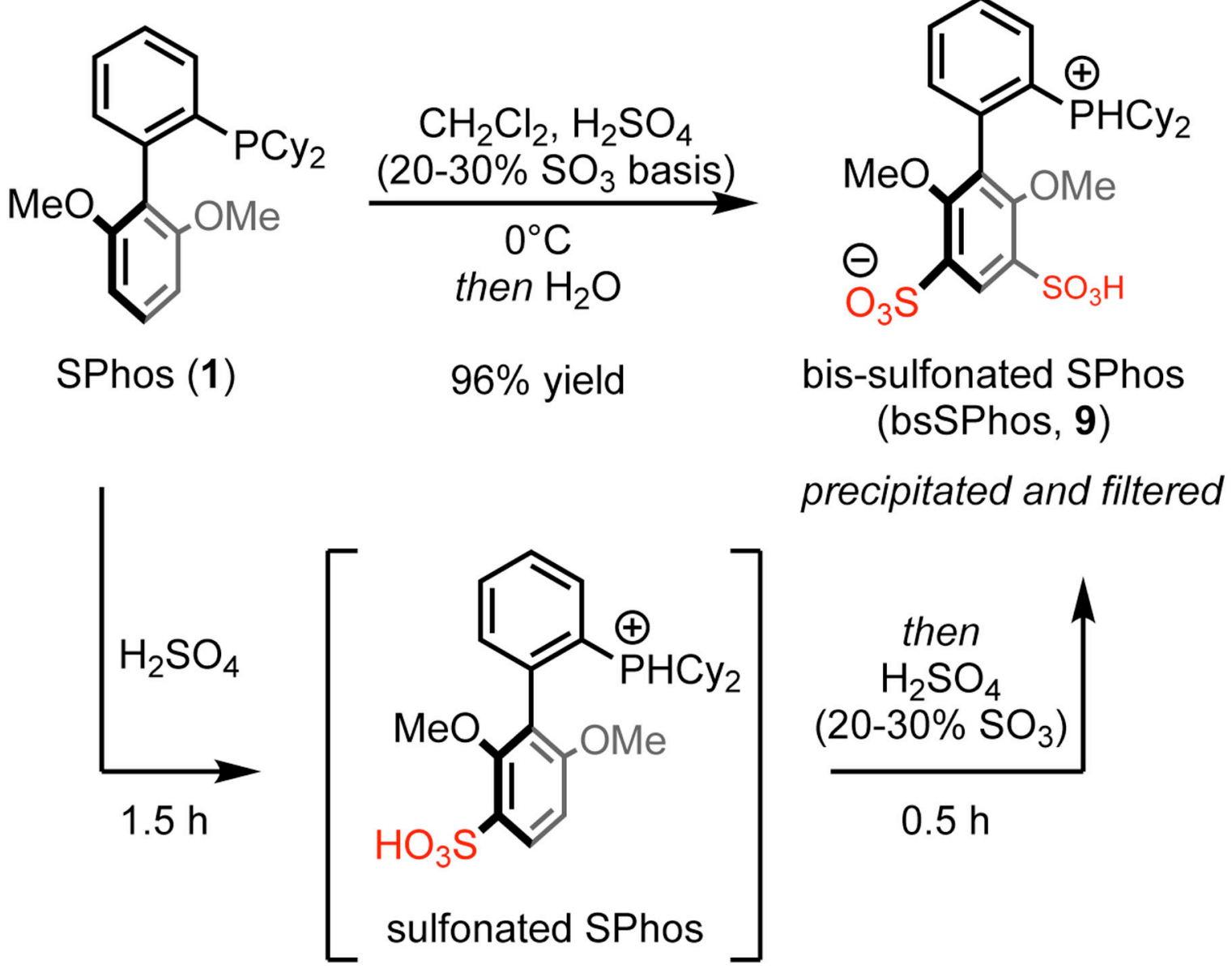

Scheme 3.

Synthesis of bis-sulfonated SPhos (bsSPhos). 
Table 1.

Optimization for a single regioisomeric product in the sulfonation of $t$-BuBrettPhos

\begin{tabular}{|c|c|c|c|}
\hline${ }_{t \text {-BuBre }}^{i \mathrm{Pr}}$ & $\begin{array}{r}\mathrm{CH}_{2} \mathrm{Cl}_{2}, \mathrm{H} \\
\text { hen } \mathrm{H}_{2} \mathrm{SO}_{4}(20 \\
\text { temperat }\end{array}$ & st-BuBrettPhos (8) & (minor isomers, 12) \\
\hline Entry & Reaction Time & Temperature $\left({ }^{\circ} \mathbf{C}\right)$ & Ratio 8:12 \\
\hline $1^{a}$ & $5 \mathrm{~min}$ & 0 & $13: 1$ \\
\hline $2^{b}$ & $5 \mathrm{~min}$ & 0 & $48: 1$ \\
\hline $3^{b}$ & $5 \mathrm{~min}$ & -10 & $82: 1$ \\
\hline $4^{b}$ & $1 \mathrm{~h}$ & -10 & $84: 1^{c}$ \\
\hline
\end{tabular}

${ }^{a}$ The reaction was carried out by the addition of fuming sulfuric acid dropwise to a solution of ligand.

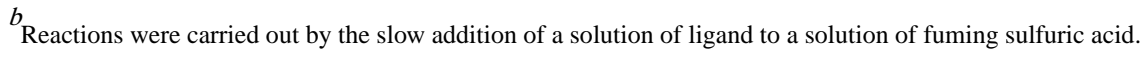

$c^{c}$ Under these conditions, an increased number of unidentified by-products were observed. 